\title{
Controllable Crack Propagation in Off-Axis Crushing of Stitched Composite Absorbers
}

\author{
N. Ghafari-Namini ${ }^{1}$, H. Ghasemnejad ${ }^{2}$ \\ ${ }^{1}$ Zodiac Aerospace, Camberley, UK \\ ${ }^{2}$ School of Aerospace \& Aircraft Engineering, Kingston University, London, UK \\ Email: hessam.ghasemnejad@kingston.ac.uk
}

Received 15 June 2015; accepted 11 September 2015; published 16 September 2015

Copyright (C) 2015 by authors and Scientific Research Publishing Inc.

This work is licensed under the Creative Commons Attribution International License (CC BY). http://creativecommons.org/licenses/by/4.0/

(c) (i) Open Access

\begin{abstract}
Crack growth resistance plays a different role in crashworthiness analysis since the progressive energy absorption is based on controllable fracture mechanisms. In this regard, the present paper studies the efficient crack growth resistance in off-axis crushing of composite tubular structures by implementing natural fiber yarns. One of the through-to-thickness reinforcement methods known as stitching has been chosen to influence the axial and off-axis crushing process. Improving the crack growth resistance and appropriate fiber breakage at different stages of crushing process can significantly improve the resistance force and consequently the energy absorption capability of composite absorbers in axial and off-axis crushing. This analysis will be applied to non-stitched and stitched CFRP composite boxes which showed brittle fracture and transverse shearing crushing modes under off-axis loading of $\mathbf{1 0}$ degrees. The analytical methods are also implemented to analysze the effect of various failure mechanisms such as bending, friction, bundle fracture, and interlaminar crack growth for the observed crushing modes. The proposed model is able to predict the crushing load and crush force efficiency in close agreement from experimental studies.
\end{abstract}

\section{Keywords}

Composite, Mechanism, Stitched, Crush, Off-Axis

\section{Introduction}

Many of composite absorbers are designed to dissipate large amounts of impact energy under axial crushing, bending or combined loading with controllable collapse manner in the collision event. Extensive research during the last decade focused on the axial impact of crashworthy components. Understanding this particular failure mode has a very high significance in the health and integrity of automotive and aircraft structures. Various re- 
searches have studied the energy absorption capability of composite tubular structures. The most recent works are reviewed as follow.

Mahdi and Hamouda [1] performed an experimental investigation of in-plane crushing of composite hexagonal ring system between platens. The rings angles are varying between $45^{\circ}$ and $70^{\circ}$. Six layers of woven roving E-glass fabric/epoxy wrapped over wooden mandrel to get thickness of about $3 \mathrm{~mm}$. Results showed that the crush failure loads and energy absorption capability were greatly affected by the ring geometry, arrangement and loading conditions. Priem et al. [2] studied crash energy absorption of 2.5D braided thermoplastic composite absorbers. According to their results the specific energy absorption increases with increasing braiding angle and decreasing length-to-diameter ratio, for glass/polypropylene tubes. On the opposite, the specific energy absorption deceases with braiding angle for carbon/polyamide tubes. Song and Du [3] investigated four types of round composite tubes, $[ \pm 75]_{3}$ glass/polyester, $[0 \pm 75]_{2}$ glass/epoxy, $[ \pm 15]_{3}$ glass/polyester and $[ \pm 15]_{3}$ glass/ epoxy under off-axis loadings to examine how the ply pattern and off-axis angle affected the energy absorption performances. More recently, Othman and Jailani [4] applied a numerical analysis to study the impact dynamic effects of crush performance of the tapered cross-section tubes, containing straight, single taper, double taper, taper triple and fourth tapered lightweight polyurethane foam filled alloy steel thin-walled tube. In their work an off-axis angle load dynamic effects in front of crash were examined at an angle of 0, 5, 10, 15 and 20 degrees. Their results showed that the energy absorption capacity had significantly affected tube with various oblique angle, impact velocity and wall thickness. It was found that the foam filling in a thin-walled tubes increased the amount of energy absorbed compared with empty tubes. Ghasemnejad and Hadavinia [5] have extensively investigated the axial and off-axis crashworthy behavior of CFRP and GFRP composite box structures. In their work, the inclination angles of $5^{\circ}, 10^{\circ}, 20^{\circ}$, and $30^{\circ}$ of loading direction with respect to composite box axis were studied experimentally under quasi-static crushing process. Due to crack propagation in Mixed-Mode I/II and more friction and bending resistance at one side of composite box which firstly contacted the crushing platen, the amount of SEA at off-axis loading of $10^{\circ}$ was the maximum in comparison with other off-axis crushing loadings. Recently they showed that natural flax fibers had the main advantage over the synthetic fibers (e.g. carbon and glass) of providing both resistance and progressive failure (effective crack growth resistance) in the wall of composite box absorbers (see Figure 1). Progressive failure can provide high energy absorption in a controllable behavior which reduces the main injuries and death during a crash event [6].

In previous studies the off-axis crushing process of tubular structures was studied based on the various parameters related to geometry, laminate design and loading conditions. However, the effective interwall crack propagation namely controllable crack propagation is another factor which can significantly influence the energy absorption capability in off-axis loading. This research studied a controlling process to reinforce the laminate through the thickness using natural fibre yarns to improve the crack resistance during the crushing process known as effective crack growth.

\section{Off-Axis Crushing Process of Non-Stitched and Stitched Crush Box}

As it was discussed before the off-axis angle of $10^{\circ}$ showed the highest energy absorption capability in comparison with other crushing angles. In this regard, this angle was chosen in this work to investigate the failure mechanisms of stitched composite plates involved in off-axis crushing process. Details of manufacturing and specimen preparation are explained in detail in [6]. The composite boxes were off-axially crushed using Universal Testing Machine with $500 \mathrm{kN}$ capacity. The specimen was tightly gripped at one end in the clamp-set by screwing up

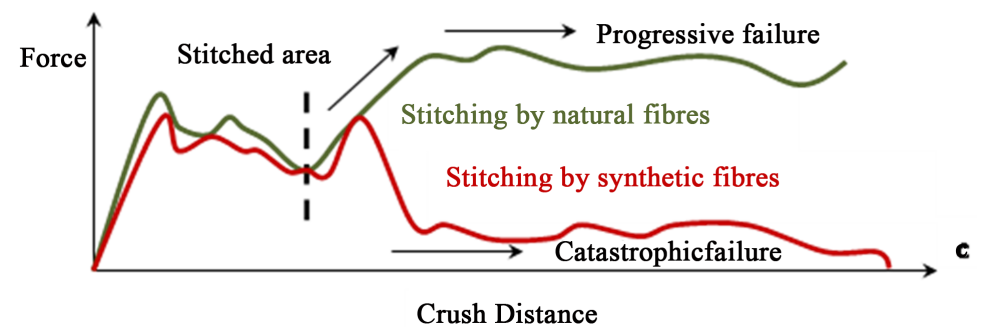

Figure 1. (a) progressive failure by natural flax fibres and (b) catastrophic failure by synthetic fibres. 
the adjustable bolt. The whole clamped fixture together with the gripped specimen was placed on the lower crushing plane testing machine and was tilted to a predetermined off axis angle with a certain spacer load cell at a vertical cross-head crushing rate of $2 \mathrm{~mm} / \mathrm{min}$ (see Figure 2). Complete compaction of tested tube and load increases sharply. Load and displacement were recorded by an automatic data acquisition system.

In contrast with axial crushing load the off-axis failure is non-symmetrically process. Load increased initially to the value of $37 \mathrm{kN}$ at the displacement of $5 \mathrm{~mm}$ (see Figure 3). Macro fragmentation was observed and material splayed to outside of the box (see Figure 4(a)). First the materials of walls 2 and 3 were splayed with inward fronds in one side and long outward fronds. As load increases, wall 4 was engaged in crush process (see Figure 4(b)). By continuing the crushing process, the longitudinal cracks advanced by splitting the box wall in different segments. As shown in Figure 4, failure starts at maximum load of $119 \mathrm{kN}$ at $15 \mathrm{~mm}$. The crushing process of $10^{\circ}$ off-axis loading was in brittle fracture mode which debris was created for the crushed specimen. The nature of derbies was somewhat different from axial loading. At this stage the load-displacement curve declined slightly. However, after a small displacement, it remains constant (see Figure 4(c) and Figure 4(d)). The extension of fracture in off-axis loaded box was greater in wall 1, similar walls 2 and 3, and less in wall 4 (see Figure 5). This comparison between force-displacement graphs (average of 3 tests for each specimen) in Figure 6 shows that stitched composite box has lower mean crushing force in comparison with non-stitched composite box. In this case, the amount of energy absorption is lower than the relevant capability in non-stitched composite box. The transverse shearing (fragmentation) crushing mode was observed for these tests. This crushing mode shows lower energy absorption capability in comparison with brittle fracture mode (see Figure 7). It is recommended to move down the position of stitched area to allow more time for bending of bundle and then the crack resistance will get started.

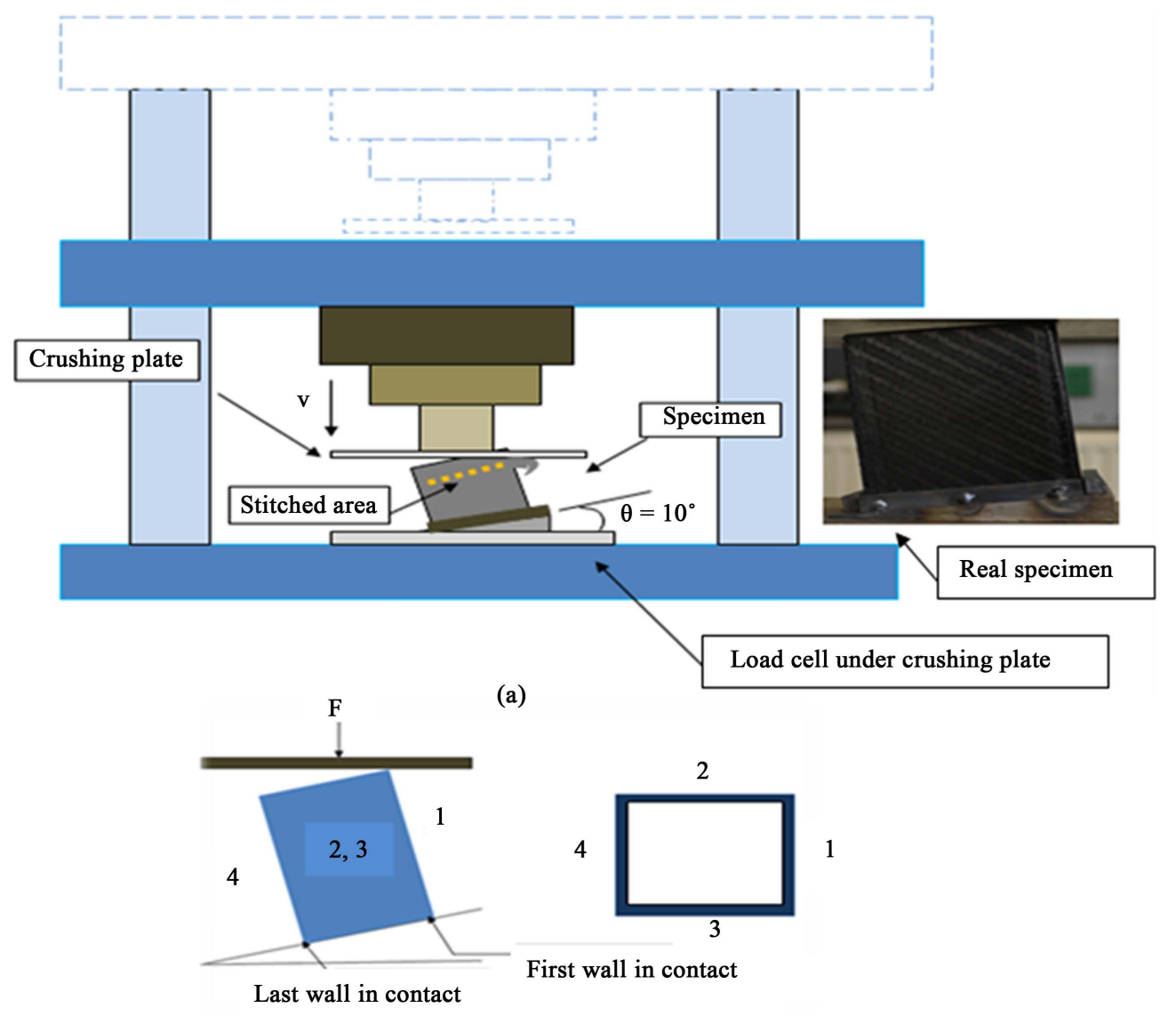

(b)

Figure 2. (a) Schematic demonstration of off-axis progressive crushing test and (b) Indication of wall numbers. 


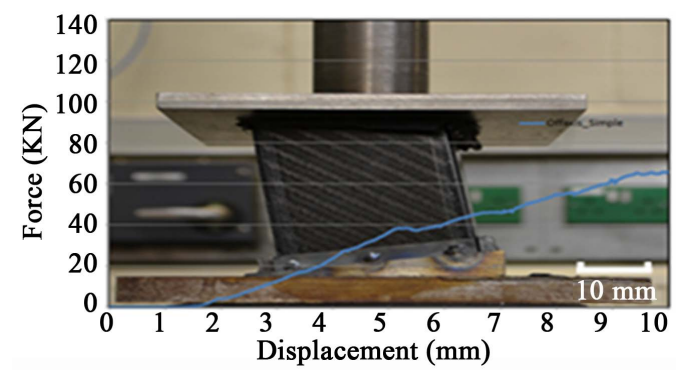

(a)

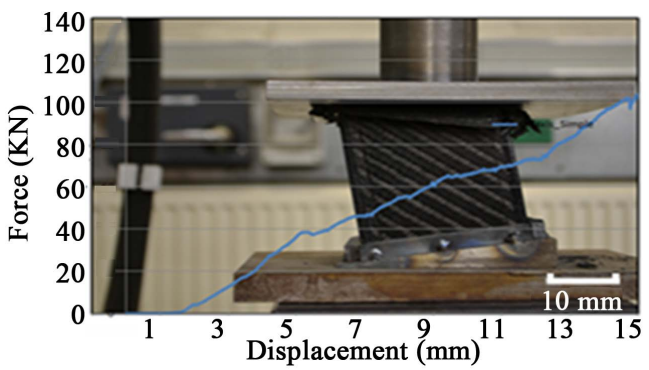

(b)

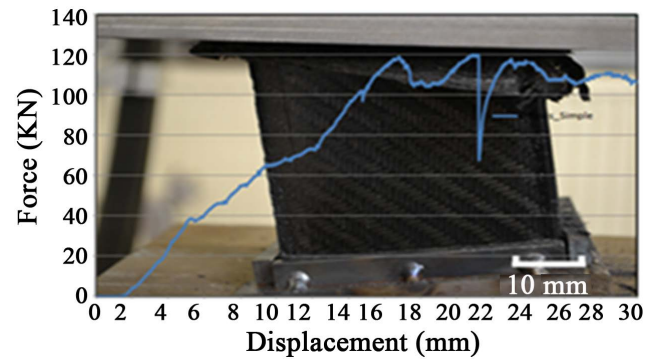

(c)

Figure 3. Force $(\mathrm{kN})$-displacement $(\mathrm{mm})$ curves and deformation history of non-stitched CFRP composite box under offaxial crushing load.
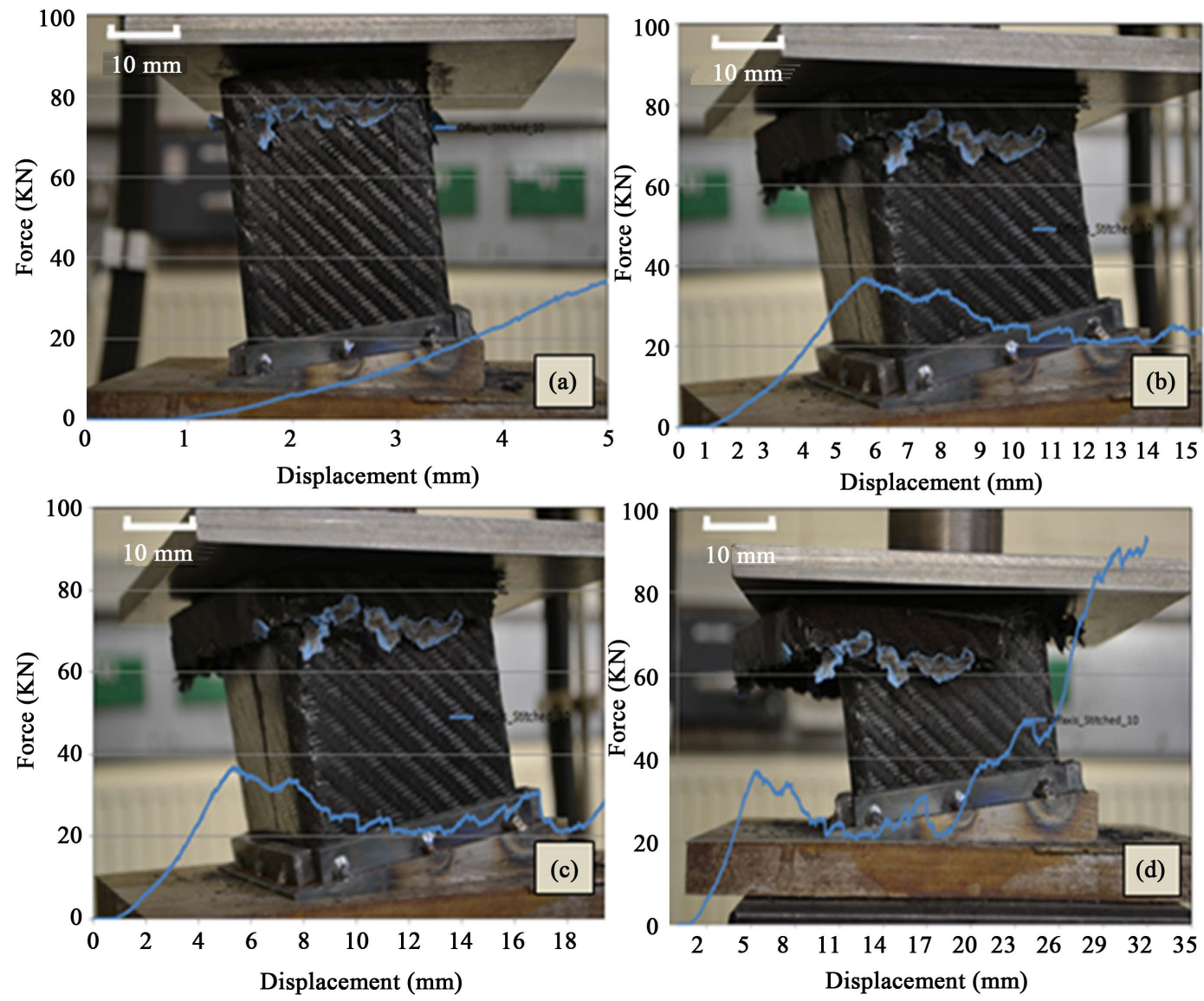

Figure 4. Force (kN)-displacement $(\mathrm{mm})$ curves and deformation history of stitched CFRP composite box under off-axial crushing load. 


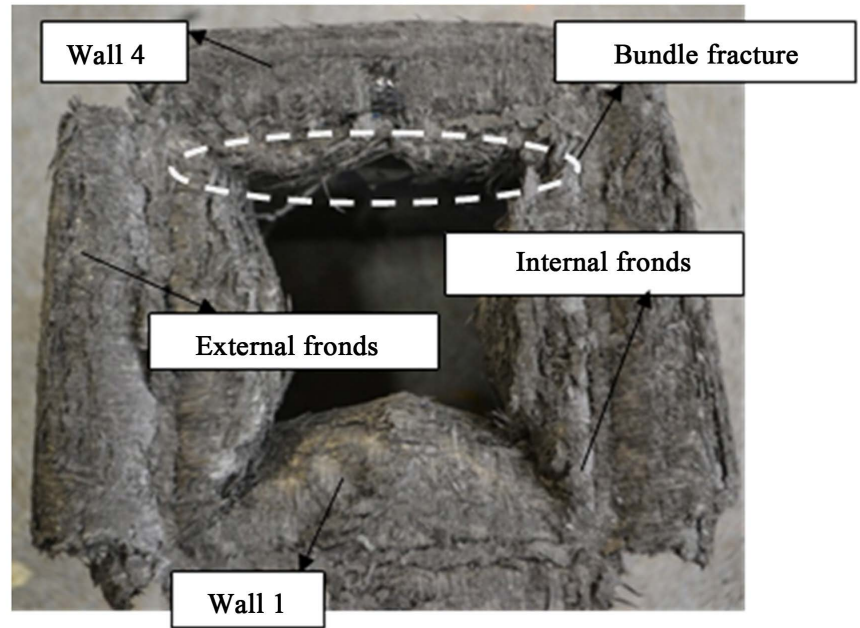

Figure 5. Top view of off-axis crushed non-stitched CFRP composite box.

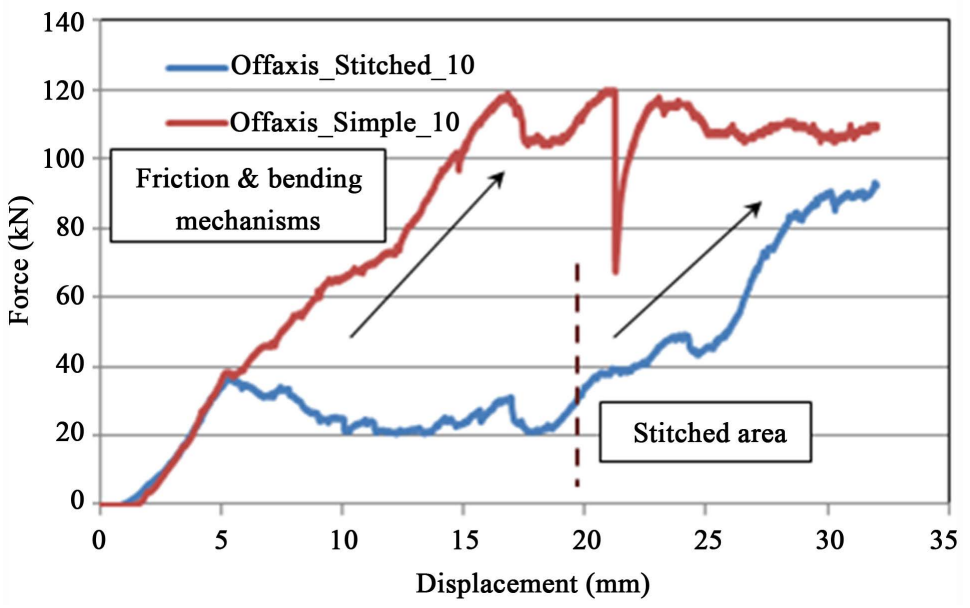

Figure 6. The comparison of force-crush distance in off axis stitched and non-stitched composite crush box.

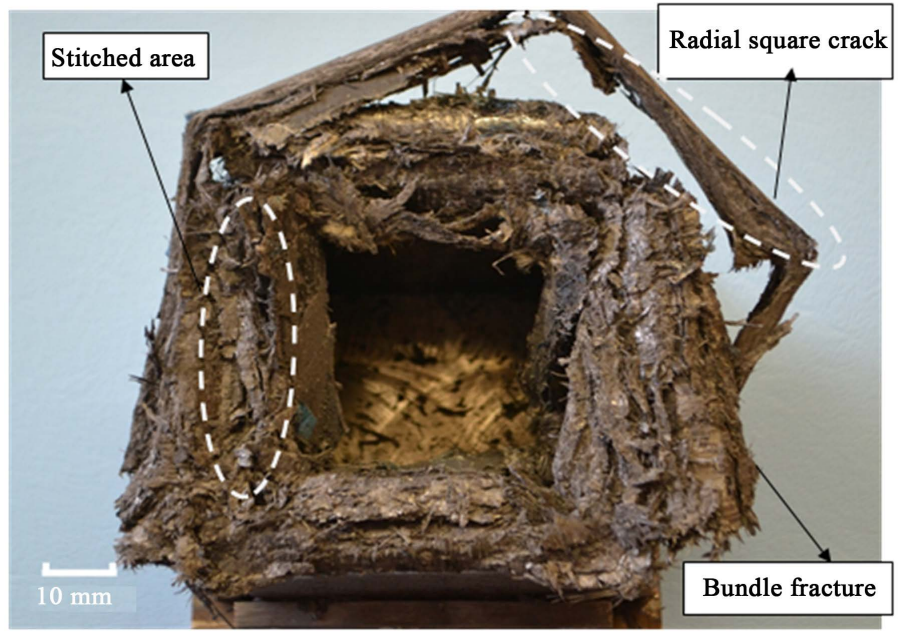

Figure 7. Top view of off-axis crushed stitched CFRP composite box in transverse shearing mode. 


\section{Analysis of Experimental Results}

In previous work of authors [6] the combination of crushing modes of lamina bending/brittle fracture and transverse shearing/local buckling were analyzed. In the current research off-axis loading caused brittle fracture crushing mode for non-stitched CFRP box while the transverse shearing (fragmentation) mode was observed for stitched composite box. In the following analytical model which is based on energy balance approach the mean crushing force, $\mathrm{F}_{\mathrm{m}}$, in off-axis crushing of square composite box is obtained in two levels of triggering stage and sustained crushing stage. The external work of crushing platen is dissipated by mechanisms of friction, bending, fracture and shear deformation. The idealised crush zone for our analytical model is shown in Figure 8.

The energy dissipated by friction between crushing platen and the debris inside the internal and external fronds in brittle fracture crushing mode is assumed into two stages, first is related to triggering stage and second one is related to sustained crushing stage and it can be obtained from,

$$
U_{f}=\left[2 \cdot \mu \cdot \frac{F}{2} \cdot x\right]_{\text {Triggering Stage }}+\left[2 \cdot \mu \cdot \frac{F}{2} \cdot x\right]_{\text {Sustained Crushing Stage }}=2 \cdot \mu \cdot x
$$

where $x=\lambda \sin \varphi, F=$ Force and $\mu=$ Coefficient of Friction . The experimentally measured coefficient of friction is $\mu=0.35$ for CFRP.

The energy dissipated by frond bending was calculated by assuming that the whole cross section of the frond will reach to the flexural bending stiffness, $\sigma_{b}$.

The flexural strength, $\sigma_{b}$, for each lay-ups of CFRP was measured $\sigma_{b}=570 \mathrm{MPa}$ from 3 PB experiment. The energy dissipated in bending for brittle fracture crushing mode at stationary hinge lines is

$$
U_{b}=\left[\int_{0}^{\frac{\pi}{2}} \frac{\sigma_{b}(4 b) t^{2} \mathrm{~d} \varphi}{8}\right]_{\text {Triggering Stage }}+\left[\int_{0}^{\frac{\pi}{2}} \frac{\sigma_{b} b t^{2} \mathrm{~d} \varphi}{8}\right]_{\text {Sustained Crushing Stage }}=\frac{5 \pi b t^{2} \sigma_{b}}{16}
$$

where $b=$ width of box and $t=$ thickness of wall,

The energy dissipated by interlaminar crack propagation in Mixed-Mode I/II is calculated from interlaminar fracture energies. In brittle fracture mode there is one interwall Mixed-Mode I/II crack at sides of the box as shown in Figure 7. Therefore,

$$
U_{d}=4 b \lambda\left(G_{I / I I C}\right)
$$

The Mixed Mode I/II interlaminar fracture toughness is measured experimentally by ADCB test. Dissipated energy by axial splitting, shear deformation and bundle fracture for brittle fracture crushing mode are calculated as follow:

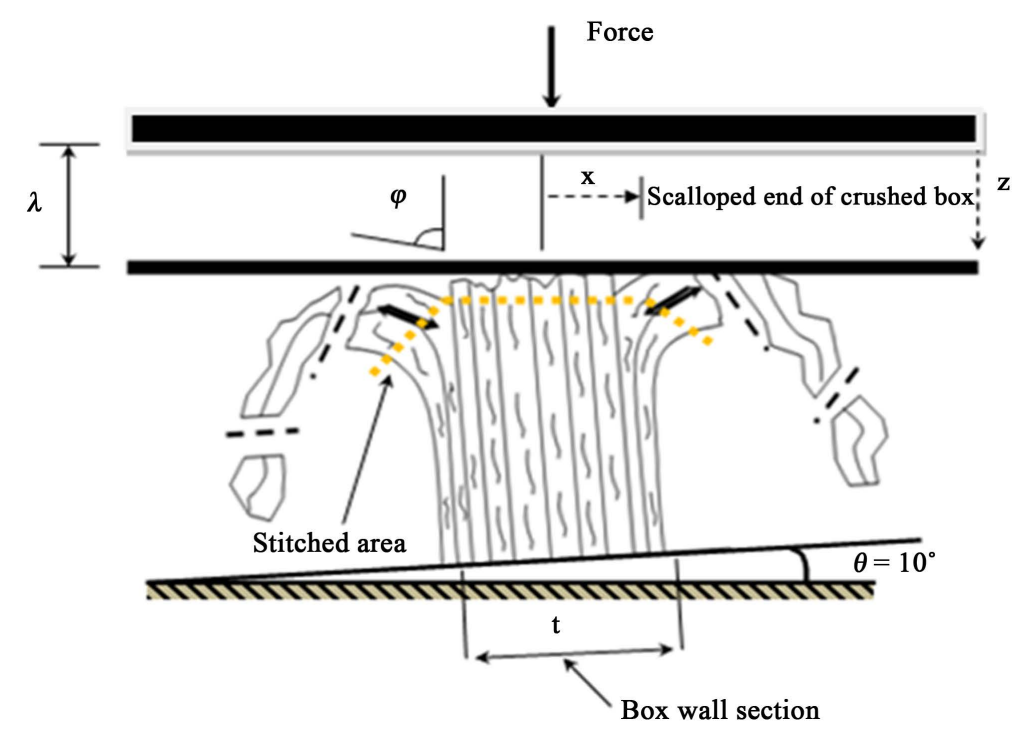

Figure 8. Ideal crush zone, stitched CFRP composite box in off-axis angle of $10^{\circ}$. 


$$
U_{c}=\frac{5 \pi t \lambda^{2} \sigma_{u}^{2}}{E_{z}}
$$

where $E_{z}$ is Young's modulus in axial direction of the crush box.

$$
\begin{gathered}
U_{s}=\frac{1}{2} \int_{V} \frac{\tau^{2}}{G_{12}} \mathrm{~d} V=\frac{2 b t \lambda \tau_{s}^{2}}{G_{12}} \\
U_{\text {bu.f }}=\frac{2.5 \pi t b^{2} \sigma_{u}^{2}}{E}
\end{gathered}
$$

where

$$
E=E_{y}, G_{12}=\text { Shear Modulus }
$$

The energy balance for the brittle fracture crushing process during a single stroke crush distance is

$$
U_{\text {External.work }}-U_{\text {Friction }}=U_{\text {Bending }}+U_{\text {Delamination }}+U_{\text {Corner.Fracture }}+U_{\text {Shear.Deformation }}+U_{\text {Bundle.Fracture }}
$$

Substituting from Equations (1) to (6) in Equation (8), the energy dissipated by brittle fracture crushing mode, $\mathrm{UBF}$, is

$$
U_{B F}=F(1-2 \mu) \lambda=\frac{5 \pi b t^{2} \sigma_{b}}{16}+4 b \lambda\left(G_{I / I I C}\right)+\frac{5 \pi t \lambda^{2} \sigma_{u}^{2}}{E_{z}}+\frac{2 b t \lambda \tau_{s}^{2}}{G_{12}}+\frac{2.5 \pi t b^{2} \sigma_{u}^{2}}{E_{y}}
$$

Therefore, the mean oscillatory crushing force in a stable brittle fracture progressive crush is:

$$
F=\frac{1}{(1-2 \mu)}\left[\frac{5 \pi b t^{2} \sigma_{b}}{16 \lambda}+4 b\left(G_{I / I C}\right)+\frac{5 \pi t \lambda \sigma_{u}^{2}}{E_{z}}+\frac{2 b t \tau_{s}^{2}}{G_{12}}+\frac{2.5 \pi t b^{2} \sigma_{u}^{2}}{E_{y} \lambda}\right]
$$

A single stroke crush distance for brittle fracture mode can be found from Equation (11) by setting $\partial F / \partial \lambda=0$

$$
\lambda=\sqrt{\frac{5 b t \sigma_{b} E_{y}+40 b^{2} \sigma_{u}^{2}}{80 \sigma_{u}^{2}} \frac{E_{z}}{E_{y}}}
$$

The energy balance for the transverse shearing crushing process during a single stroke crush distance is

$$
U_{\text {External.work }}-U_{\text {Friction }}=U_{\text {Bending }}+U_{\text {Corner.Fracture }}+U_{\text {Shear.Deformation }}+U_{\text {Bundle.Fracture }}
$$

Substituting from Equations (1), (2), (3), (5), (6) and (7) in Equation (12), the energy dissipated by transverse shearing crushing mode, UTS, is

$$
U_{L B}=F(1-\mu) \lambda=\frac{\pi b t^{2} \sigma_{b}}{4}+\frac{5 \pi t \lambda^{2} \sigma_{u}^{2}}{E_{z}}+\frac{2 b t \lambda \tau_{s}^{2}}{G_{12}}+\frac{0.31 \pi t b^{2} \sigma_{u}^{2}}{E_{z}}
$$

Furthermore, the mean oscillatory crushing force in a stable transverse shearing progressive crush is:

$$
F=\frac{1}{(1-\mu)}\left[\frac{\pi b t^{2} \sigma_{b}}{4 \lambda}+\frac{5 \pi t \lambda \sigma_{u}^{2}}{E_{z}}+\frac{2 b t \tau_{s}^{2}}{G_{12}}+\frac{2.5 \pi t b^{2} \sigma_{u}^{2}}{E_{z} \lambda}\right]
$$

A single stroke crush distance for transverse shearing mode can be found from Equation (15) by setting $\partial F / \partial \lambda=0$

$$
\lambda=\sqrt{\frac{b t \sigma_{b} E_{z}+10 b^{2} \sigma_{u}^{2}}{20 \sigma_{u}^{2}}}
$$

The mean force during the progressive crushing can be found by calculating $\lambda$ from Equations (11) and (15) substituting in Equations (10) and (14) (see Table 1). The results were in good agreement with the relevant experimental results and the discrepancy between experimental and theoretical results is less than $1.5 \%$ and $5 \%$ for brittle fracture and transverse shearing crushing modes, respectively. 
Table 1. Comparison of experimental and analytical mean force results of off-axis CFRP composite box.

\begin{tabular}{ccccccc}
\hline Off-axis specimen & Cushing failure mode & $\mathrm{F}_{\mathrm{m}}$ (Exp.) kN & SEA (Exp.) kJ/kg & $\mathrm{F}_{\mathrm{m}}$ (Anal.) kN & SEA (Anal.) kJ/kg & Error (\%) \\
\hline Stitched & Transverse Shearing (TS) & 57 & 12.5 & 54 & 11.8 & 5 \\
Non-stitched & Brittle Fracture (BF) & 118 & 25.8 & 116 & 25.4 & 1.5 \\
\hline
\end{tabular}

\section{Conclusion}

In this paper experimental analysis was performed to study the effect of stitching on controllable crack growth in off-axis crushing behaviour and energy absorption of carbon/epoxy twill-weave composite box composite. The two types of specimens, stitched and non-stitched composite boxes, were subjected to quasi-static off-axial compression load. Two fracture mechanisms of bundle fracture and interlaminar crack propagation in MixedMode I/II were found for all composite boxes at off-axis crushing process. Owing to crack propagation in Mixed-Mode I/II and more friction and bending resistance at one side of the composite box, which first contacted the crushing platen, the amount of SEA at off-axis loading of $10^{\circ}$ was higher than the equivalent value in axial loading. However, the CFE of non-stitched composite boxes at off-axis loading is higher than the CFE of stitched boxes. It was observed that in off-axis loading the energy absorption of stitched boxes was less than the energy absorption of non-stitched boxes. The main problem was related to the position of stitched area in the box wall. It is assumed that lower position from top of the box could allow all sides of box firstly get involved in crush process, and then the stitched area increases the load resistance and consequently energy absorption capabilities. Various mechanisms based on corner fracture, interlaminar fracture, friction, bending and shear deformation were performed to predict the off-axis crushing mean force and CFE of composite absorbers. The analytical method was also carried out to study the mechanisms involved in crushing process which could show good agreement with the relevant experimental results.

\section{References}

[1] Mahdi, H. and Hamouda, A.M.S. (2012) Energy Absorption Capability of Composite Hexagonal Ring Systems. Materials \& Design, 34, 201-210. http://dx.doi.org/10.1016/j.matdes.2011.07.070

[2] Priem, C., Othman, R., Rozycki, P. and Guillon, O. (2014) Experimental Investigation of the Crash Energy Absorption of 2.5D-Braided Thermoplastic Composite Tubes. Composite Structures, 116, 814-826. http://dx.doi.org/10.1016/j.compstruct.2014.05.037

[3] Song, H.-W. and Du, X.-W. (2002) Off-Axis Crushing of GFRP Tubes. Composite Science and Technology, 62, 20652073. http://dx.doi.org/10.1016/S0266-3538(02)00152-5

[4] Othman, A. and Jailani, A. (2014) Off-Axis Crushes Simulation of Thin-Walled Tapered Tubes Inserted with FoamFilled Structures. APCBEE Procedia, 9, 395-400. http://dx.doi.org/10.1016/j.apcbee.2014.01.069

[5] Ghasemnejad, H. and Hadavinia, H. (2010) Off-Axis Crashworthiness Characteristic of Woven Glass/Epoxy Composite Box Structures. Journal of Reinforced Plastics and Composites, 29, 2306-2330. http://dx.doi.org/10.1177/0731684409347807

[6] Ghafari-Namini, N. and Ghasemnejad, H. (2012) Effect of Natural Stitched Composites on the Crashworthiness of Box Structures. Materials \& Design, 39, 484-494. http://dx.doi.org/10.1016/j.matdes.2012.03.025 